\title{
A Case Report of Aerococcus urinae Prosthetic Valve Infective En- docarditis Initially Misidentify
}

Natalia Lattanzio, $M D^{1^{*}}\left(\mathbb{D}\right.$, Qassem Abdelal, MD ${ }^{1}$, Stephen Bell, MD ${ }^{1}$ (D) Talal Alkayali, MD ${ }^{1}$, Christian Lorenzo, $M D^{1}$, Ricardo Villasmil, $M D^{1}$, Wilhelmine Wiese-Rometsch, $M^{1}$ and Manuel Gordillo, $M D^{2}$

${ }^{1}$ Internal Medicine, Sarasota Memorial Hospital, USA

${ }^{2}$ Infectious Disease, Sarasota Memorial Hospital, USA

*Corresponding author: Natalia Lattanzio, MD, Internal Medicine, Sarasota Memorial Hospital, $1700 \mathrm{~S}$

Tamiami Trail, Sarasota, FL 34239, USA, Tel: (954)-494-9397

\begin{abstract}
Infective endocarditis (IE) is most commonly caused by species such as staphylococci, streptococcus and enterococci. When IE is caused by organisms such as Aerococcus urinae, misidentification of the organism in culture is common, resulting in misdiagnosis. We present a case of IE caused by Aerococcus urinae in a 67 -year-old male with a bioprosthetic aortic valve whose culture initially grew micrococcus.
\end{abstract}

\section{Abbreviations}

ALT: Alanine Transaminase; AST: Aspartate Transaminase; ALP: Alkaline Phosphatase

\section{Introduction}

Aerococcus urinae is a gram-positive catalase negative cocci that has been recognized as part of the normal flora of the urinary tract. The incidence of it causing a urinary tract infection (UTI) is less than $0.8 \%$ and even less of invasive disease [1]. Due to its similarity in morphology to other species such as streptococcus and staphylococcus, $A$. urinae tends to be underdiagnosed or misdiagnosed. It has also been associated with culture-negative infective endocarditis [2]. A. urinae IE should always be suspected in patients with pre-existing urinary tract pathologies; especially in the presence of a bioprosthetic valve. We describe a case of $A$. urinae prosthetic vale endocarditis (PVE) in a patient that had recently undergone prostatic photovaporization.

\section{Case Report}

A 67-year-old male presented to the emergency de- partment (ED) complaining of intermittent fevers, chills, diaphoresis, generalized malaise, confusion, aphasia and dyspnea on exertion for three days prior to presentation. His past medical history was significant for bioprosthetic aortic valve replacement 7 years ago secondary to the congenital bicuspid valve, benign prostatic hyperplasia with outlet obstruction and recurrent urinary tract infections (UTI). Six weeks prior to presentation, he underwent photovaporization of his prostate complicated by 3 episodes of UTI. A urine culture failed to show significant growth but a commercial urine polymerase chain reaction (PCR) yielded Aerococcus urinae, and the patient began treatment with sulfamethoxazole/ trimethoprim. Despite treatment with antibiotics, he developed fever with bouts of confusion and transient visual disturbances. Further evaluation by an infectious disease specialist yielded a diastolic cardiac murmur in the background of a bioprosthetic valve, raising concern for a complicated bioprosthetic valve endocarditis. At the time, the urine PCR results of Aerococcus urinae UTI were not available and the patient was referred to ED for further evaluation.

At presentation, physical exam findings consisted of a heart rate of 90 , blood pressure $124 / 86$, temperature of $103{ }^{\circ} \mathrm{F}$ and respiratory rate of 24 . Bilateral coarse breathing sounds were appreciated in addition to a soft systolic murmur with a superimposed diastolic component at the left upper parasternal border. There were no peripheral signs of infective endocarditis (IE) such as Janeway lesions or splinter hemorrhages. Laboratory

Citation: Lattanzio N, Abdelal Q, Bell S, Alkayali T, Lorenzo C, et al. (2020) A Case Report of Aerococcus urinae Prosthetic Valve Infective Endocarditis Initially Misidentify. Int J Clin Cardiol 7:184. doi. org/10.23937/2378-2951/1410184

Accepted: June 16, 2020; Published: June 18, 2020

Copyright: (c) 2020 Lattanzio N, et al. This is an open-access article distributed under the terms of the Creative Commons Attribution License, which permits unrestricted use, distribution, and reproduction in any medium, provided the original author and source are credited. 

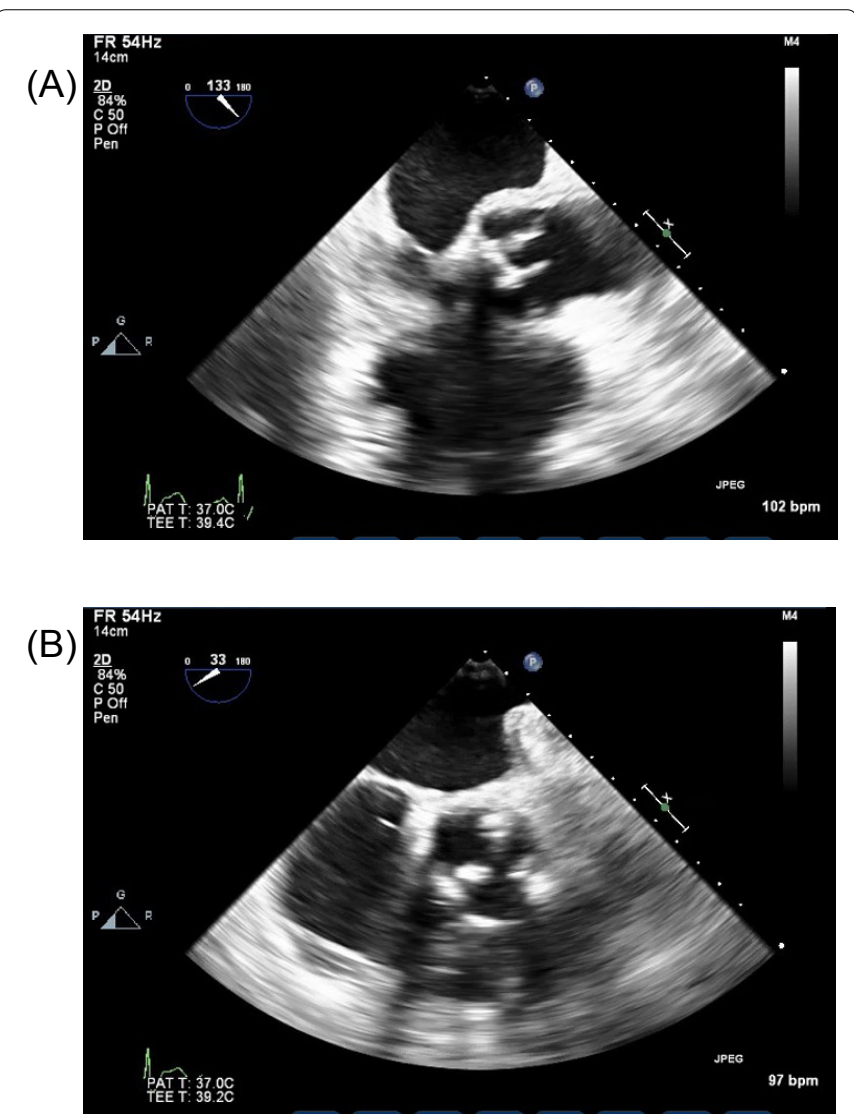

Figure 1: (A) Transesophageal echocardiogram (TEE) mid-esophageal view showing mobile echo density on the prosthetic aortic valve. (B) Transesophageal echocardiogram (TEE) transgastric to mid-esophageal view showing prosthetic aortic valve with thickened leaflets suggestive of endocarditis.

data was significant for a leukocytosis of $12,300 / \mathrm{uL}$ with left shift, ALP 337 U/L, AST 144 U/L, ALT 207 U/L. Urinalysis revealed moderate leukocytes, nitrates negative, large blood and more than 100 leukocytes.

The patient was admitted and further evaluated by cardiology and infectious disease specialists. A transesophageal echocardiogram (Figure $1 \mathrm{~A}$ and Figure 1B) was significant for thickening leaflets of the aortic bioprosthetic valve with a small mobile lesion and moderate-severe regurgitation concerning for IE. Empiric antibiotic therapy with vancomycin and cefepime was initiated in conjunction with cardiothoracic surgery consultation. The patient underwent a redo aortic valve debridement and replacement. Surgical intervention revealed a small tunnel of abscesses. Blood cultures, urine cultures and one out of two surgical cultures remained negative. The second surgical specimen was significant for neutrophilic predominance and cultures eventually grew micrococcus, a misidentification. In light of this, the surgical sample was sent for 165 ribosomal broad-spectrum PCR which was positive for Aerococcus urinae. He was given ertapenem for a total of 6 weeks with no evidence of recurrence upon follow up.

\section{Discussion}

Aerococcus urinae is an aerobic, alpha-hemolytic, gram-positive coccus that is usually arranged in tetrads but can also be found in pairs or clusters. Due to these features, it can be easily be misidentified as staphylococcus or streptococcus $[3,4]$. Its mechanism of virulence is similar to that of streptococci viridans in that it causes platelet aggregation and biofilm formation. Biofilm formation makes patients with prosthetic heart valves particularly susceptible to $A$. urinae PVE. According to literature reviews and research, the prevalence of A. urinae endocarditis is 3 per every million people [1]. The presentation is similar to PVE secondary to other etiologies. The gold standard for accurate identification of $A$. urinae is via $16 \mathrm{~s}$ ribosomal RNA gene sequencing [1]. But due to it being time consuming and costly, this method of diagnosis is not frequently performed. To our knowledge this is the first reported case established by non-culture diagnosis, with urine infection demonstrated by PCR and valvular tissue 16S RNA testing yielding the etiologic cause. This organism tends to be highly responsive to penicillin/cephalosporins and resistant to sulfonamides and aminoglycosides [2]. Due to it being often underdiagnosed, it is important to keep $A$. urinae as a differential diagnosis in the event of IE in a patient with pre-existing urinary tract pathologies.

\section{Conflicts of Interest}

All authors listed have contributed equally to the making of this manuscript and all authors report no conflict of interest.

\section{References}

1. Harsha Tathireddy, Sahitya Settypalli, John J Farrell (2017) A rare case of aerococcus urinae infective endocarditis. J Community Hosp Intern Med Perspect 7: 126-129.

2. Adeel M, Tariq $S$, Akthar $\mathrm{H}$, Zaghloul $A$, lorgoveanu $\mathrm{C}$, et al. (2017) Case Report: A rare case of prosthetic valve infective endocarditis caused by Aerococcus urinae. F1000Res 6: 1998.

3. Sierra-Hoffman M, Watkins K, Jinadatha C, Fader R, Carpenter JL, et al. (2005) Clinical significance of Aerococcus urinae: A retrospective review. Diagn Microbiol Infect Dis 53: 289-292.

4. Yabes JM, Perdikis S, Graham DB, Markelz A (2018) A rare case of Aerococcus urinae infective endocarditis in an atypically young male: Case report and review of the literature. BMC Infect Dis 18: 522.

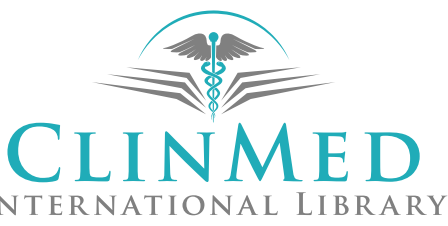

